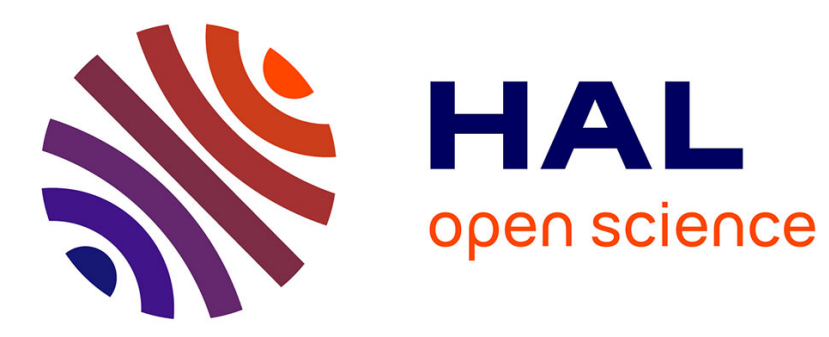

\title{
Le dispositif masochiste
}

\author{
Julie Mazaleigue-Labaste
}

\section{To cite this version:}

Julie Mazaleigue-Labaste. Le dispositif masochiste. La Clinique lacanienne, 2016, 28 (2), pp.119. halshs-01989507

\section{HAL Id: halshs-01989507 https://shs.hal.science/halshs-01989507}

Submitted on 22 Jan 2019

HAL is a multi-disciplinary open access archive for the deposit and dissemination of scientific research documents, whether they are published or not. The documents may come from teaching and research institutions in France or abroad, or from public or private research centers.
L'archive ouverte pluridisciplinaire HAL, est destinée au dépôt et à la diffusion de documents scientifiques de niveau recherche, publiés ou non, émanant des établissements d'enseignement et de recherche français ou étrangers, des laboratoires publics ou privés. 


\section{Le dispositif masochiste}

Version auteur avant corrections de Mazaleigue-Labaste, 2016. « Le dispositif masochiste », La clinique lacanienne 28 (2016), p. 119-136

Comme l'ont montré les historiens queer à partir des cas de la Grèce antique et de Rome, il existe des modalités historiques d'organisation de l'expérience érotique : des pratiques, mais aussi du désir et des fantasmes ${ }^{1}$. Je propose ainsi une thèse forte à partir d'une lecture politique du masochisme: bien loin d'être une réalité psychique et comportementale transhistorique, le masochisme érotique est une expérience propre à notre modernité politique dont il intègre les structures. Le vieux constat de Theodor Reik étant toujours valable (pourquoi diable un masochiste satisfait réclamerait-il une analyse ?) ${ }^{2}$, je ferai appel à l'analyse conceptuelle, à l'histoire, aux théories sur le masochisme, aux textes littéraires et aux témoignages.

Récuser la transhistoricité du masochisme, c'est immédiatement dire que le point de vue sexologique, celui de la description des pratiques sexuelles et du désir dont elles peuvent être l'objet (toujours présent en filigrane dans l'appréhension psychanalytique du masochisme érotique) laisse échapper le phénomène masochiste, ses variations et ses métamorphoses. Car ce qui conduit deux sujets à poser deux actes comportementalement indiscernables - piquer, fouetter, couper, frapper - peut radicalement différer. Le même geste infligeant une grande douleur peut-être posé par un bourreau mandaté par l'Etat, par un sadique, comme par ce que Gilles Deleuze nommait le «bourreau masochiste $»^{3}$, celui ou celle occupant la fonction de dominant.e, de « maître » ou de «maîtresse » dans le dispositif scénographie masochiste. Si ce dernier emprunte bien au sadisme les apparats de la cruauté physique et morale, il les investit d'un sens et d'une fonction tout à fait distincts. A l'inverse, le point de vue sexologique, englué dans la fascination pour les actes ${ }^{4}$, ne cesse de nous ramener aux vieilles

1. Voir notamment David Halperin, 1989/2000. Cent ans d'homosexualité et autres essais sur l'amour grec, Paris : EPEL.

2. Théodor Reik, 1941/2000. Le masochisme, Paris : Payot.

3. Gilles Deleuze, 1967/2007. Présentation de Sacher-Masoch. Le froid et le cruel, Paris : Editions de Minuit.

4. Ainsi cet analyste qui, face à une patiente fortement dépressive et par ailleurs pratiquante du BDSM en tant que soumise, ne cessait, lors d'une cure entreprise dans le cadre d'une hospitalisation en clinique, de la ramener 
lunes du «plaisir dans la douleur », de la complémentarité du sadisme et du masochisme, ou de la « forme érotique de la haine ». Il s'agit donc de se départir de cette tendance.

Dans les limites de cet essai, je laisserai de côté la question des relations entre masochisme, rapports de genre et orientations sexuelles. Au délà des métamorphoses de la seconde moitié du $19^{\mathrm{e}}$ siècle (le masochisme érotique était fortement hétéronormé, et lié aux inégalités sociales et politiques entre hommes et femmes ${ }^{5}$ ) jusqu'aux communautés cuir nordaméricaines au $20^{\mathrm{e}}$ siècle (qui ont fait l'objet des analyses de l'anthropologue Gayle Rubin ${ }^{6}$ ), à la scène lesbienne Leatherdyke (notamment objet de travaux de Lynda $\operatorname{Hart}^{7}$ ) et à la problématisation de l'empowerment par les pratiques $\mathrm{BDSM}^{8}$, un continuum du phénomène masochiste est envisageable si l'on tient compte de structures politiques plus fondamentales, et transversales aux rapports de genre.

\section{Le sadomasochisme n'existe pas}

Il faut définitivement se défaire d'une idée issue de la tradition psychopathologique, transmise à la sexologie et encore persistante dans le champ analytique freudien ${ }^{9}$ : la complémentarité entre sadisme et masochisme fondée sur l'idée d'un investissement érotique de la douleur, qui légitimerait la pertinence et l'usage du concept de « sadomasochisme ». Ce dernier relève en réalité d'une structuration spécifiquement masochiste de l'imaginaire, dans laquelle la douleur n'endosse qu'une fonction dérivée. Cette structure imaginaire prend la forme privilégiée d'une scène, qui forme un dispositif aux fonctions et aux finalités précises que j'explorerai. Dans le théâtre masochiste - dont les Cérémonies de femmes de Jeanne de Berg sont la

\footnotetext{
à sa pratique du bondage, du fouet et de la sodomie lorsqu'elle parlait de sa mère (dépressive, sourde, agressive et constituant un fardeau tout à fait objectif) et du deuil, à faire pour l'une et l'autre, du père mort (témoignage). 5. Toute l'œuvre de Léopold von Sacher-Masoch est traversée par ces thèmes pourtant négligés, il apparaît de manière évidente dans La Venus à la Fourrure. Léopold von Sacher-Masoch, Leopold, 1967/1870, La Vénus à la Fourrure, Paris : Éditions de Minuit. Voir aussi Leopold von Sacher-Masoch, 2011. La Madone à la fourrure, postface de Jean Allouch, Paris : EPEL. Le fantasme sado-masochisme tel qu'il s'est structuré au 19e siècle était fortement lié à la domination masculine. Le contât est lucide chez Masoch. Ainsi, la Vénus à la fourrure se termine sur une analyse politique et sociale des sources du masochisme : "La femme, telle que la nature l'a créée et telle qu'elle attire l'homme aujourd'hui, est son ennemi et ne peut être pour lui qu'une esclave ou un tyran, mais jamais une compagne. Elle ne pourra l'être que lorsqu'elle lui sera égale en droits et qu'elle le vaudra par sa formation et son travail. Pour le moment nous n'avons qu'une alternative : être le marteau ou l'enclume».

6. Pour une anthologie de ses textes, voir Gayle Rubin, 2011. Deviations. A Gayle Rubin Reader, Duke University Press

7. En particulier Lynda Hart, 1998. Between the Body and the Flesh, New York : Columbia University Press.

8. Acronyme de Bondage, Domination, Soumission, Masochisme.

9. En témoigne le concept de «monade sadomasochiste » forgé par le psychanalyste italien Franco de Masi, qui exprime au plus haut degré cette complémentarité. Franco de Masi, date. La perversion sadomasochiste, Paris : Editions d'Ithaque.
} 
parfaite illustration ${ }^{10}$ - se meuvent des personnages incarnant des fonctions spécifiques du dispositif ; mais le sadique n'y a pas sa place.

De cette non complémentarité du sadisme et du masochisme témoignent des histoires aux temporalités distinctes. Certes, les deux catégories furent inventées au même moment, en 1890, durant l'Âge d'Or de la psychopathologie sexuelle par ce pape des perversions que fut Richard von Krafft-Ebing, en référence aux littératures du Marquis de Sade et de Leopold von Sacher-Masoch ${ }^{11}$. Point essentiel : ces références fondatrices sont fictionnelles. C'est le romanesque qui donne son nom à ce qui relevait encore pour l'esprit médical de l'époque du domaine de la pathologie mentale. On sait aujourd'hui le rôle central de la littérature et des légendes historiques dans la naissance des savoirs sur les sexualités dites perverses ${ }^{12}$ et l'imprégnation de la clinique psychosexuelle par la fiction - de Gilles de Rais à Sade en passant par le Rousseau des Confessions, qui venait exemplifier le fétichisme chez Alfred Binet ${ }^{13}$. Sadisme comme masochisme ne sauraient être appréhendés hors d'un imaginaire érotique originairement construit dans et par la fiction.

Mais cette naissance conjointe des terminologies psychiatriques n'épuise pas l'historicité des phénomènes. Il semble que l'histoire du sadisme s'inscrive sur la toile de fond des rapports entre volupté et cruauté qui imposèrent une "nouvelle texture du plaisir ${ }^{14}$ aux corps et aux esprits d'Europe de l'Ouest à partir du $16^{\mathrm{e}}$ siècle, et qu'elle soit celle de la Vénus ouverte, retracée par Georges Didi-Huberman à partir du Quattrocento ${ }^{15}$. La Vénus ouverte est l'envers, au sens propre, de la Vénus parfaite de Botticelli : la femme dépecée par les chiens, éventrée, cette ouverture la ravalant au rang de pur corps en dévoilant ses organes - son anatomie dans ce qu'elle a de plus objectif. Comme le rappelle Didi-Huberman, Sade fut fasciné par les Vénus anatomiques du musée de la Specola de Florence, ces cires médicales mettant en scène des femmes dans des poses lascives, le corps ouvert, les organes finement sculptés visibles au regard de tous. Cette fascination du Marquis surprendra peu. Un des motifs dominant son œuvre est l'exploration des orifices dans la douleur jusqu'à la création de

10. Jeanne de Berg (pseud. de Catherine Robbe-Grillet), date. Cérémonies de femmes, Paris : Grasset.

11. Le terme «sadisme » existait en réalité dans la langue française depuis les années 1830, pour qualifier à la fois les cruautés analogues à celles que l'on attribuait aux Marquis et le « système antinaturel » constitué par son oeuvre.

12. Vernon Rosario, 1997/2000. L'irrésistible ascension du pervers, entre littérature et psychiatrie, Paris : EPEL ; Julie Mazaleigue-Labaste, 2014. Les déséquilibres de l'amour. La genèse du concept de perversion sexuelle de la révolution française à Freud, Paris ; Editions d'Ithaque.

13. Alfred Binet, 1887/2001. Le fétichisme dans l'amour, Paris : Payot.

14. Robert Muchembled, 2005. L'orgasme et l'Occident, Paris : Seuil.

15. Georges Didi-Huberman, 1999. Ouvrir Vénus, Paris : Gallimard. Pour une analyse plus développée, voir Julie Mazaleigue-Labaste, op. cit., p. 157 sq. 
nouvelles ouvertures, le découpage anatomique du corps, le corps désiré non dans sa complétude mais dans la juxtaposition de ses parties - la bouche, le con, le cul ${ }^{16}$. Cet imaginaire de la cruauté fouaillant les corps fut central dans l'invention des «perversions sexuelles » modernes en 1849, en France, à la suite de l'affaire criminelle du sergent nécrophile François Bertrand, dit le « vampire de Montparnasse ${ }^{17}$. Nécrophile, il ne l'était pas seulement. Bertrand était surtout celui qui éventrait et démembrait les corps féminins. Et à cette occasion, quelques décennies avant que Krafft-Ebing ne nomme le sadisme, les psychiatres en inventèrent le concept, «l'amour sanglant»- dont un des modèles explicite était l'œuvre sadienne.

Si le sadisme est bien dominé par la figure de la Vénus ouverte, il ne peut être ni le complément, ni le symétrique du masochisme. Pourquoi alors cette erreur ? Parce que le phénomène masochiste a ressaisi des éléments qui appartiennent à l'histoire plus ancienne du sadisme. Au $18^{\mathrm{e}}$ siècle, un nouveau type d'érotisation de la cruauté a triomphé dans la littérature. A la cruauté physique s'est adjointe la cruauté morale. Nonnes violentées dans les couvents et servantes dominées par leurs maîtres ont rejoint la Vénus ouverte, jusqu'au triomphe de la «cruauté des petits maîtres » (l'expression est des frères Goncourt), dont Les liaisons dangereuses est une figure exemplaire. La cruauté pouvait désormais se passer de l'effraction violente des frontières du corps : ce qui est érotique, c'est la cruauté affective et morale qui témoigne des relations de pouvoir entre des hommes qui dominent et des femmes dupes et donc victimes (sauf une, Merteuil, mais dont on sait la fin tragique). On peut lire l'œuvre de Sade comme un moment charnière dans l'histoire de la violence érotisée, un lieu où se combinèrent le fantasme ancien de la Vénus ouverte et la jouissance nouvelle de la cruauté affective infligée à une femme innocente - voici Justine, vertueuse infortunée qui meurt traversée par la foudre de la bouche au $\operatorname{con}^{18}$.

Mais l'érotique proprement masochiste, elle, émergea au $19^{\mathrm{e}}$ siècle. Son expression exemplaire est l'œuvre de Sacher-Masoch, au plus au degré sa célèbre Vénus à la Fourrure. Cette Vénus n'est ni la fille ni la sœur de la Vénus ouverte. Ce qu'elle représente, Foucault

16. Octave Mirbeau ne l'oubliera pas dans son Jardin des supplices, en reprenant à Sade une torture qui deviendra un des nombreux «supplices chinois » poussant les raffinements de la cruauté à leur paroxysme : le supplice du rat se frayant un chemin sanglant dans l'anus du supplicié ... Octave Mirbeau, 1899. Le jardin des supplices, Paris : Charpentier-Fasquelle.

17. Julie Mazaleigue-Labaste, op. cit., p. 127-163.

18. Donatien Alphonse François de Sade, 1801/1987. Histoire de Juliette, ou les prospérités du vice, tome 2, parties IV à VI, Paris : Pauvert. 
l'avait bien perçu ${ }^{19}$, c'est l'érotisation d'un rapport de domination, c'est-à-dire d'une relation de pouvoir entre un dominant et un dominé, élément essentiel de la scène masochiste l'acronyme contemporain BDSM (Bondage, Domination, Soumission Masochisme) étant à ce titre bien plus pertinent que « sadomasochisme ». Or, à l'inverse du sadique qui désire réduire le sujet à ce qui est en lui de moins subjectif - sa viande, ses tripes -, toute relation de pouvoir suppose le maintien de la subjectivité des deux membres du rapport. Ceci n'échappa pas à Krafft-Ebing. Il fit certes l'erreur d'affirmer la relative complémentarité entre sadisme et du masochisme, mais perçut clairement le cœur de ce dernier : la soumission, qui peut à la limite se passer de tout recours aux pompes de la cruauté physique ${ }^{20}$. On comprend mieux pourquoi Séverin, héros et narrateur de La Vénus à la fourrure, ne cesse de poursuivre un plaisir « suprasensuel », au delà du physique. Et le moment durant lequel il vit de manière la plus pure son expérience masochiste est expurgé de toute douleur et de tout exercice de la cruauté : ayant porté au bain Wanda, sa maîtresse devenu Maîtresse dont il $s$ 'est fait, à $s a$ demande, le valet, il a contemple dans un moment de calme absolu, instant suprasensuel d'où toute excitation sexuelle a disparu.

Que le sadisme exige la cruauté et l'effraction violente des frontières du corps et de l'esprit, soit. Mais le masochisme n'a que faire de l'impossible « plaisir dans la douleur » qui obséda les savants de la fin du $19^{\mathrm{e}}$ siècle, chimère conceptuelle à laquelle Freud se laissa prendre lorsqu'il en fit l'orientation passive d'une pulsion de cruauté et d'agression sexuelle dont la forme active serait le sadisme. La faute à l'acharnée volonté scientifique de l'époque de trouver in fine un fondement biologique aux phénomènes psychiques, qui conduisit Albert von Schrenck-Notzing à inventer «l'algolagnie» passive (le masochisme) et active (le sadisme $)^{21}$ en invoquant un hypothétique phénomène physiologique de synesthésie de la douleur et du plaisir. La faute aussi à l'obsession d'une description sexologique la plus large possible des comportements sexuels déviants, mouvement duquel Freud resta tributaire - en témoignent les notes de bas de page du premier des Trois Essais ${ }^{22}$. Par l'analyse fine des

19. «L'idée que le $\mathrm{S} / \mathrm{M}$ est lié à une violence profonde, que sa pratique est un moyen de libérer cette violence, de donner libre cours à l'agression est une idée stupide [...] On peut dire que le S/M est l'érotisation du pouvoir, l'érotisation de rapports stratégiques. » Michel Foucault, 1982. « Michel Foucault, une interview : sexe, pouvoir et la politique de l'identité » (entretien avec B. Gallagher et A. Wilson, Toronto, juin 1982 ; trad. F. DurandBogaert), dans Michel Foucault, Dits et Ecrits, Tome IV (1980-1988), Paris : Gallimard, texte n³58, p. 735-746. 20. Richard von Krafft-Ebing (von), 1893/1895. Psychopathia Sexualis, avec des recherches spéciales sur l'inversion sexuelle, traduction de la huitième édition allemande par Émile Laurent et Sigismond Csapo, Paris : Carré, p. 121-122.

21. Albert von Schrenck-Notzing, 1892. Die Suggestions-Therapie bei krankhaften Erscheinungen des Geschlechtssinnes : mit besonderer Berücksichtigung der konträren Sexualempfindung, Stuttgart : Enke.

22. Sigmund Freud, 1905d/1987. Trois Essais sur la théorie sexuelle, trad. française, OCF.P VI, 2006 
textes de Sade et Masoch, Gilles Deleuze parvint à cette même conclusion d'une erreur freudienne : la relation dite "sadomasochiste" ne met pas en scène un sadique et un masochiste, mais un, et même une, bourreau masochiste, initiée et fétichisée par l'homme soumis, répondant à la demande de ce dernier, comme Wanda renonçant finalement à être la maîtresse de Séverin pour apprendre à devenir sa Maîtresse.

Pourtant, n'y a-t-il pas des cas de masochisme, extrêmes mais révélateurs, qui nous propulsent dans la recherche de la douleur pour elle même? Ainsi l'homme décrit par Michel de $\mathrm{M}^{\prime} \mathrm{Uzan}^{23}$ dont le corps entier portait les traces de tortures qu'il exigeait de ses «maîtres » mandatés pour l'occasion, et dont on se demande bien par quel maléfice il put les supporter. Ici encore, l'extrême des actes ne doit pas fasciner le regard. M’Uzan souligne que ce masochiste hors-normes ne cessait d'instrumentaliser ses bourreaux, qu'il initiait et poussait jusque dans leurs retranchements au point d'en faire fuir certain par ses folles demandes. On pourrait dire, dans une perspective lacanienne, que ce masochiste cherchait à susciter de l'angoisse : chez ses bourreaux mais aussi chez le tiers, ici M'Uzan, à qui il se confia. Mais ce désir d'angoisse de l'Autre ne peut-il pas être réintroduit dans le dispositif masochiste par lequel la relation de pouvoir se trouve érotisée? Car l'homme soumis, y compris chez Masoch, n'est dépossédé de son pouvoir que sur la scène. Le pouvoir de la Maîtresse (ou du Maître) n'est conféré que par sa place dans le dispositif et ne s'exerce qu'au sein de ce dernier : ses effets peuvent, certes, être crûment réels, mais il reste un pouvoir imaginaire. Et si le personnage soumis est dominé, l'individu qui l'incarne, lui, reste le détenteur réel d'un pouvoir tout aussi réel. C'est à cette condition qu'il peut initier son bourreau, voire le forcer à endosser cette fonction - comme Séverin face à Wanda. Lorsque la Maîtresse dessillée s'émancipe de son rôle en tombant amoureuse d'un autre homme, son prétendu esclave n'hésite pas à la violenter - brutal retour du réel, qu'accusa aussi cette dominatrice professionnelle des années 2000 lorsqu'elle découvrit qu'aucun de ses esclaves à la scène ne lui ferait la politesse de venir la chercher à la ville lors d'un voyage ${ }^{24}$. Et quand Séverin occupe réellement la place qu'il pensait être l'apogée de son fantasme - être dominé par un autre homme, tiers qui serait à la fois l'amant et l'instrument de sa maîtresse - il déchante immédiatement : ni Wanda ni son amant grec ne jouent le jeu masochiste, le désir tombe, les fastes du théâtre laissent place au vaudeville. En guise de suprasensuel ne restent que la colère

23. Michel de M’Uzan, 1972/1983, «Un cas de masochisme pervers. Esquisse d'une théorie », dans De l'art à la mort, Paris : Gallimard, p. 125-150.

24. Témoignage. 
et l'humiliation sociale du cocu encombrant et rossé par son rival devant son ancienne maîtresse, qui se réjouit de ce tour bien mérité.

\section{La structure contractuelle du masochisme}

La structure masochiste ne se caractérise toutefois pas seulement comme une érotisation de relations de pouvoir ; elle intègre un trait structurel qui dépend du cadre politique général régulant les relations humaines dans nos sociétés libérales et démocratiques : le contractualisme.

La nécessité du contrat masochiste a été analysée par Gilles Deleuze à partir de l'œuvre de Masoch $^{25}$. Il serait erroné de n'y voir qu'un dispositif purement littéraire. Sans même prendre en compte les contrats que Masoch signa - et fit signer - dans sa vie à ses amantes, le contrat reste aujourd'hui structurant pour les relations BDSM, qu'il se présente sous une forme écrite aux clauses développées et explicitées (comme le contrat signé par Salomé et Mastermind, couple phare du milieu BDSM hétérosexuel français dans les années 2000) ou relève d'un accord oral entre les partenaires. La fonction du contrat dans le dispositif masochiste est architectonique : il fixe l'extension et les limites du cadre spatial et temporel de la relation dominant/dominé, les prérogatives du dominant, et les modalités et les limites de l'exercice du pouvoir qui lui est conféré. Le safeword, ce signe-avertissement du dominé défini par un accord préalable entre les partenaires, dont la fonction est d'indiquer le franchissement des limites par le dominant (en particulier des limites des capacités physiques), en est un parfait exemple.

Mais cette structure contractuelle du masochisme fait de ce dernier un phénomène intrinsèquement libéral, au sens du libéralisme politique et économique. Le contrat est en effet une forme encadrant des échanges et fixant les obligations deux parties, qui exige l'égalité en droit et en droits des contractants (Wanda et Séverin, tous deux de haute naissance, sont des égaux) et leur consentement éclairé. Pour cette raison, le contrat masochiste n'a non seulement aucun intérêt, mais surtout aucun sens dans une société où l'esclavage est réel - Séverin et Wanda, qui formulent le projet d'un voyage en Turquie pour mettre en pratique les fantasmes du premier, y renoncent immédiatement : on ne peut signer un contrat par lequel se réalise l'imaginaire de l'esclavage là où l'esclavage est une réalité politique. La structure du fantasme masochiste ne peut pas davantage se déployer dans une société aux principes 
politiques fondamentalement inégalitaires (pensons à l'Ancien Régime en France, ou aux sociétés de castes) : une telle société ne repose pas sur l'idée d'un pacte social qui engagerait chacun de ses membres considérés comme des sujets volontaires aptes à passer une convention et à en respecter les obligations. C'est dire que le masochisme ne peut pas être un invariant transhistorique et transculturel, car il est intrinsèquement liée à l'histoire politique de nos sociétés : c'est une érotique essentiellement démocratique et libérale.

Que permet cette structure contractuelle du masochisme? Elle ouvre l'espace d'un jeu sur les relations de pouvoir. Le dispositif masochiste doit être une structuration imaginaire et exige une scène, un théâtre, des personnages, car il faut que les individus qui entrent en relation restent au fond, et réellement, des sujets éthiques, juridiques et politiques pour passer le contrat qui rend possible la relation. Rappelons l'énergie avec laquelle Rousseau, grand analyste de la forme contractuelle, expliquait justement l'impossibilité de tout «pacte de soumission » réel, et encore moins de «pacte d'esclavage » - soulignant ironiquement que je ne peux passer contrat avec mon esclave, car on ne peut contracter avec une de ses propriétés : ceci conduirait à l'absurdité d'un contrat avec soi-même ${ }^{26}$. D'où l'impression que le masochisme, "c'est du chiqué » - bien évidemment, c'est du chiqué, cela doit même l'être. D'où le caractère stéréotypique des rôles, des scenarii, la récurrence des gestes, des pratiques et des témoignages de soumission (le dominé à genoux devant le dominant, le soumis léchant les chaussures de la Maîtresse, etc.). Car dans ce jeu sur le pouvoir, les individus doivent s'effacer derrière la fonction qu'ils endossent, désormais incarnée par le personnage. Les éléments du théâtre BDSM jouent comme éléments de dé-singularisation au profit du dispositif - les rôles, le cadre. D'où, aussi, la déception toujours possible pour les acteurs, voire même l'impression de ridicule qui peut se dégager lorsque les scènes fantasmées ou décrites par la littérature sont mises en pratique - le ridicule qui s'impose lors d'une soirée «Histoire d'Ô» pourtant soigneusement organisée dans un pavillon de province, ou le désappointement de cet homme rêvant de longues années de participer à une des Cérémonies de Jeanne de Berg et mesurant, le grand jour arrivé, l'écart entre fantasme et réalité ${ }^{27}$. C'est que le réel résiste toujours à l'imaginaire qui l'excède. Mais ce serait faire erreur que d'en rester à ce constat apparemment négatif.

Car le dispositif masochiste, parce qu'il est intrinsèquement contractuel, rend par là impossible toute défection ou ravalement réel de la subjectivité à la viande ou à la chose. Il

26. Jean-Jacques Rousseau, 1762/2001. Du Contrat Social ou Principes du droit politique (Livre I), Paris : Flammarion.

27. Témoignages. 
exige le consentement mutuel (norme contemporaine cardinale de la sexualité) de partenaires considérés comme égaux - au moins idéalement - et par là la préservation de l'intégrité éthique, juridique et politique de l'individu ${ }^{28}$. C'est alors dire que le dispositif masochiste s'oppose frontalement à la violence et tend même à la rendre impossible en lui substituant le jeu des relations de pouvoir. Les pratiques de contrainte (attaches, bondage, carcans), l'usage de la douleur voire l'effraction du corps (plugs vaginaux et anaux, dont certains aux dimensions impressionnantes; aiguilles; suspensions par crochets « hameçons » passant au travers de la peau et reliés à des cordes), même extrêmes, ne sont pas cruelles : elles ont pour fonction d'être les signes et les effets de ce pouvoir sur soi délégué par un individu à un autre au travers du contrat. A l'opposé de la violence (et en particulier de la violence sadique), le dispositif masochiste cherche au contraire à assurer que le rapport des corps soit une relation intersubjective, protégée et idéalement garantie par le contrat et ses clauses. Équilibre complexe, en raison des pratiques BDSM elles-mêmes - un dérapage reste toujours possible mais surtout de l'absence d'instance supérieure, transcendante aux acteurs, qui garantirait la bonne application des accords consensuels. Mais à ce problème, les acteurs répondent. D'abord par l'exigence (martelée) de la confiance entre partenaires qui pallie l'absence d'institution et joue le rôle de garantie affective du respect du contrat. Ensuite, puisque le BDSM est désormais un phénomène social, par une dynamique de formation, d'information et de pédagogie (largement renforcée par Internet, mais aussi présente dans la mise en place d' « ateliers BDSM » visant à diffuser une information auprès du public, ou encore dans les initiatives individuelles de pratiquants aguerris organisant des évènements au cours desquels ils jouent le rôle de guide et de garant du respect des limites de chacun) et la volonté d'élaborer des formes possibles de régulations immanentes partagées par les membres de la communauté - le site français BDSM ou abus est ainsi dédié aux questions que soulèvent le franchissement possible des limites du cadre contractuel vers la violence, et aux réponses à y apporter.

\section{L'espace de la folie}

Mais faut aller plus loin. Ce n'est pas seulement l'intersubjectivité des relations que garantit le dispositif masochiste. Il rend aussi possible une sorte de folie, en un sens précis : des formes d'expérience devenues inacceptables dans le cadre de notre économie affective

28. Rappelons que Gayle Rubin, érige le consentement en unique critère de pénalisation des actes sexuels. 
dominée par la figure de l'autonomie individuelle, qui sont la perte de contrôle de soi, l'extase, et l'amour fou.

L'exigence d'autocontrôle, qui relève d'une histoire longue magistralement retracée par Norbert Elias $^{29}$, est le propre de nos sociétés libérales-démocratiques. Elle renvoie à la capacité du sujet, comme sujet psychologique, sujet de droit et personne morale, à contrôler ses actions, mais aussi ses intentions - y compris ses désirs. Loin d'y échapper, l'expérience érotique la manifeste de manière exemplaire. Déjà visible dans la modération dans les plaisirs attendue des hommes et des femmes du début du $19^{\text {e }}$ siècle (l'intempérance les faisant basculer dans les « perversions de l'instinct sexuel»-nymphomanie, satyriasis, folie érotique pour l'époque), cette exigence triomphe dans l'injonction à l'autonomie pesant sur l'individu contemporain, qui est censé contrôler l'intensité et l'expression de ses préférences érotiques, y compris celles qui sont considérées comme déviantes. Il bascule sinon dans une dépendance qui fait de lui un « sex addict» ou un «paraphile troublé » à soigner - rappelons que dans le DSM 5, un des critères cardinaux qui distingue la paraphilie, simple expression d'une variété sexuelle, du trouble paraphilique (trouble mental) est la «détresse psychique » issue de l'incapacité d'exercer un contrôle sur ses désirs, ses fantasmes et/ou ses actes ${ }^{30}$.

Or le dispositif masochiste permet pour le dominé d'échapper à ces exigences lourdes pesant sur l'individu - celles-là même qui induisent des dépressions ${ }^{31}$ - tout en les respectant parfaitement. Car la scène BDSM est le cadre contrôlé d'une expérience physique et affective qui peut être puissante justement parce qu'elle est contrôlée. La distribution rigide des fonctions dans le dispositif crée en effet une asymétrie complète. Au dominant est dévolue la fonction du contrôle total de l'expérience, d'où ses qualités exigées qui en font l'incarnation des idéaux de l'individu contemporain - maîtrise de soi, sens des responsabilités, calme, expérience, savoir-faire - et les mises en garde de la communauté à l'encontre des prises de substance psychoactives, drogues ou alcool. La contrepartie pour le dominé, de la littérature aux témoignages en passant par les enquêtes de terrain, s'exprime de manière identique : c'est la perte du contrôle de soi contrôlée par un autre. Comme le souligne le sociologue Gilles Chantraine, actuellement engagé dans une étude de longue haleine sur le monde social du BDSM hétérosexuel en France, l’individu dominé peut en effet «lâcher prise» (termes

29. Norbert Elias, 1939/2003. La civilisation des mours, Paris : Pocket.

30. La distinction entre paraphilies et troubles paraphiliques a été introduite dans la cinquième version du Manuel diagnostique et statistique des troubles mentaux de 2013. Américaine de Psychiatrie, 2013. Manuel diagnostique et statistique des troubles mentaux (DSM) (5 $5^{\mathrm{ème}}$ éd.), Arlington (VA), Association Américaine de Psychiatrie.

31. Alain Ehrenberg, 2011. La fatigue d'être soi. Dépression et société, Paris : Odile Jacob. 
récurrents des pratiquants), c'est-à-dire déroger à ce qu'exige de lui le monde contemporain ${ }^{32}$. A l'indépendance se substituent la complète dépendance au dominant, à la volonté l'abandon aux décisions de l'autre, à l'autonomie l'hérétonomie - au sens propre, kantien : c'est un autre qui me donne ma loi. Et à la maîtrise de soi, la possibilité d'un dépassement de soi dans des pratiques du corps autrement impossibles et celle d'un désordre des émotions, des orgasmes extrêmes à la plongée dans le « subspace » - terme par lequel les anglo-saxons qualifient l'état de conscience modifié du dominé, effet des pratiques auxquelles il est soumis. Le subspace, ce n'est rien moins que l'extase. Voilà ce qu'offre le dispositif masochiste : la possibilité d'une extase dans un monde sans mystique, où ceux qui prétendent l'être, mystiques, sont des fous. Produite par les effets de contrainte et de douleur d'un dominant, cette extase n'est-elle pas une transgression magistrale de l'impératif au «bien-être » - cette marque affective de l'individu censé être autonome (et capable de "prendre soin de lui-même ») qui a renvoyé l'antique bonheur aux oubliettes?

Mais le dispositif masochiste produit d'autres émotions. Puisqu'il appelle la confiance des partenaires, l'abandon de l'un à l'autre, la responsabilité de l'un sur l'autre, il fabrique de l'amitié, des interactions sociales, de la communauté - c'est évident dans les études de Rubin sur le milieu SM gay cuir. Mais, et c'est le point sur lequel j'insisterai, il rend aussi possible l'amour fou, qui n'est désormais acceptable que dans les conditions d'une expérience contrôlée. Du savant à l'ordinaire, les affects auparavant liés à l'amour romantique sont de plus en plus disqualifiés et requalifiés en troubles psychiques, car ils mettent en péril l'autonomie et l'indépendance de l'individu - le sentiment amoureux intense devient « obsession » ou « fixette », le grand chagrin d'amour se mue en « deuil pathologique » s'il dure, la «dépendance affective» est pointée du doigt comme facteur d'entrave au développement individuel. Il faut aimer, certes, mais tout en restant soi. Penser qu'on peut mourir d'amour, voire désirer mourir d'amour relève, au mieux, d'un pur effet de discours lié à l'inexpérience de la grande jeunesse (« ce sont des mots, cela passera »), au pire, du registre du trouble affectif et pathologique. Ce fut pourtant un topos de la littérature et de la poésie de

32. «La réintroduction, sous forme de jeu, d'une asymétrie contractualisée et d'une érotisation des rapports de pouvoir, permet aussi, pour un « soumis », de « lâcher prise », au moins le temps d'un jeu érotique, autrement dit d'être délesté du poids des contraintes sociales qui pèsent sur lui en tant qu'individu. C'est l'un des paradoxes constitutif du jeu BDSM, où un individu se sent « libre » et « vrai » lorsque paradoxalement il choisit d'obéir à quelqu'un qu'il respecte, de s'en remettre à lui. C'est un peu un pied de nez aux rapports de domination réels. Parce qu'il exige d'expliciter ce qui dans la relation reste souvent implicite dans d'autres formes de relations et de sexualités, le jeu BDSM est relativement ouvert : il peut autant reproduire que troubler, atténuer ou renverser les rapports de domination traditionnels entre hommes et femmes. » Interview de Gilles Chantraine dans Les Inrocks, « Pourquoi 'Cinquante nuances de Grey' rencontre-t-il un tel succès », 22 février 2015. 
l'Eros : non seulement un affect possible mais valorisé, une preuve et une épreuve de la réalité du sentiment et de la vérité de ses expressions. Ce n'est pas seulement que l'«on considère cela comme » un trouble aujourd'hui, de manière purement extérieure, comme si la méchante psychologie tentait de nous priver de la richesse native de notre vie morale à coups de catégorisations bancales. C'est effectivement devenu un trouble dans l'économie affective qui nous caractérise ${ }^{33}$. Il faut tout de même « avoir un problème » ou « un grain » pour désirer mourir d'amour pour un autre ${ }^{34}$. Mourir d'amour, au figuré comme au propre, n'est plus une épreuve de la subjectivité mais un accident. C'est la nécessaire contrepartie du régime d'autonomie : la disqualification radicale des émotions violentes et potentiellement destructrices, au point de presque les évincer de notre univers moral normal ${ }^{35}$. Mais ce qui vaut pour l'extase dans le dispositif masochiste vaut aussi pour l'amour fou. C'est l'amour absolu que ressent, un moment, Séverin pour Wanda au bain. C'est Ô chez Pauline Réage ${ }^{36}$. C'est Sabatil chez Masoch, qui accepte de mourir sur la croix pour et par Mardona, la froide, pieuse et belle «Mère de Dieu $»^{37}$. C'est l'amour exprimé dans les larmes pour le ou la dominant.e par le ou la dominé.e rompu.e, qui se manifeste jusqu'au sein des relations économico-sexuelles entre les dominatrices professionnelles et leurs clients. Jean Streff rapporte ainsi le témoignage d'une dominatrice à laquelle un client régulier demanda la mort, comme une expression extrême et folle de l'amour qu'il portait, à ce moment précis, à la Maîtresse $^{38}$. Car le dispositif masochiste dépouille de l'individualité - réalité sociale, soumise aux multiples exigences de l'autonomie - pour faire advenir le sujet : «j'ai fait de toi un homme », dit Myriam à son époux Sabbathai Zewy, lorsqu'elle s'unit à lui, le dépouillant de ses prétentions messianiques après lui avoir fait subir la torture d'un désir dévorant ${ }^{39}$. Non un sujet abstrait - le sujet de droit qui passe convention-, mais une réalité entièrement incarnée.

33. Toute cette analyse doit beaucoup aux travaux de Pierre-Henri Castel sur l'histoire de l'obsessionnalité en Occident. Pierre-Henri Castel, 2011. Ames scrupuleuses, vies d'angoisse, tristes obsédés. Vol. I : Obsessions et contrainte intérieure, de l'Antiquité à Freud, Paris : Éditions d'Ithaque ; Pierre-Henri Castel, 2012. La Fin des coupables, suivi de Le Cas Paramord, Paris : Éditions d'Ithaque.

34. Et la forme contemporaine du « chagrin d'amour » n'a plus l'épaisseur morale qu'elle a pu avoir ; le chagrin d'amour se vit aujourd'hui sur le mode de la dépression, c'est-à-dire du déficit, qui doit être temporaire et dont on doit se remettre, comme on se remet d'une maladie (« sortez », « voyez des amis », « faites du sport »!).

35. Je ne m'érige pas ici en contemptrice d'un quelconque « homme grégaire ». La position moraliste est facile, mais fallacieuse. Car en contrepartie, notre incapacité à l'amour fou et au «mourir d'amour» est ce qui nous permet de tisser des relations interindividuelles mutuellement gratifiantes pour les partenaires, sur les modes du partage des intérêts et des goûts, de la camaraderie, de l'amitié auxquels s'ajoutent les échanges sexuels. Car c'est bien ce contrôle de la violence des affects et de leur expression qui permet la vie agréable.

36. Pauline Réage (pseud. de Dominique Aury), 1954/1999. Histoire d'Ô, Paris : Le Livre de Poche.

37. Leopold von Sacher-Masoch, 1880/1991. La Mère de Dieu, Paris : Champ Vallon.

38. Jean Streff, 2002. Les extravagances du désir. Témoignages, Paris : La Musardine.

39. Léopold von Sacher-Masoch, « Sabbathai Zewy », dans La Czarine noire et autres contes sur la flagellation, p. 353-389, Paris : Carrington, 1907. 
Il me semble que c'est ainsi que l'on peut lire les divers usages des pratiques corporelles dans le BDSM, en maintenant l'idée d'un continuum masochiste contre l'idée d'une incommensurabilité entre « léger» et « hard», hétéros et homos, gays et lesbiennes. Car s’ils sont des instruments de la relation de pouvoir et sont en ce sens dérivés de cette dernière, les usages du corps partagent un point commun : contraindre le sujet à se défaire des oripeaux de l'individualité sociale pour s'incarner.

\section{Conclusion}

Le masochisme érotique doit être appréhendé comme un dispositif spécifique sont la structure est directement liée à l'organisation sociale et politique de nos sociétés libéralesdémocratiques. La mise en scène des relations de pouvoir dans un cadre contractuel qui le caractérise s'oppose frontalement au sadisme, car il éloigne le spectre de la violence et garantit l'intersubjectivité d'une relation entre partenaires consentants et en principe égaux. Le cadre posé, il autorise pour le partenaire dominé le vécu d'une expérience érotique en rupture avec les exigences pesant sur l'individu contemporain : dépendance, perte de contrôle de soi, émotions intenses voire extrêmes.

Le dispositif masochiste induit par là une suspension de l'individu au profit du sujet. Il s'agit bien, en ce sens précis, d'une forme de subversion. Mais c'est parce qu'il est fortement canalisé et canalisant (du fantasme aux pratiques) qu'il peut produire des expériences et des émotions puissantes. Il ne met nullement en péril les valeurs et les normes cardinales qui régulent les interactions dans nos sociétés, qui sont même ses éléments structurants contractualisme, idéal d'égalité démocratique, refus de la violence, intégration de l'exigence au contrôle de soi sous la forme d'une distribution des positions ${ }^{40}$. On comprend alors mieux la raison de son succès social et culturel, des sympathiques « ateliers BDSM » à Fifty shades of $\mathrm{Grey}^{41}$.

40. Je crois qu'une analyse analogue pourrait rendre raison des divergences, toujours vivaces dans le champ des sexual studies, des gender studies et des études féministes sur le caractère politiquement subversif (chez Gayle Rubin, Pat Califia, ou Lynda Hart) ou hautement conservateur des rapports de genre dans le BDSM.

41. E.L. James, 2011/2012. Fifty Shades of Grey, Vintage Books. 